\title{
A New Energy Flux Model in the DSMC-IP Method for Nonequilibrium Flows
}

\author{
Wen-Lan Wang* and Iain D. Boyd ${ }^{\dagger}$ \\ Department of Aerospace Engineering \\ University of Michigan, Ann Arbor, MI 48109
}

\begin{abstract}
A new energy flux model is proposed for use in the Information Preservation (IP) method. The primary objective of the present study is to relax the local thermal equilibrium assumption in the previous Information Preservation method. The new IP model is constructed by assuming that the real molecules represented by a simulation particle follow a Maxwellian distribution defined by the preserved velocity and temperature. The local velocity distribution function in a cell is then assumed to be the arithmetic average of all the Maxwellian distributions within the cell. Energy flux across a cell interface can therefore be evaluated analytically. Simulations of normal shock waves over a wide range of Mach number and in different gases are performed as examples. The results show that the density predicted by the new model is in very good agreement with other numerical methods and experimental measurements, whereas temperature and velocity are only in fair agreement.

\section{Nomenclature}

a Speed of sound

$\bar{c} \quad$ Average thermal speed, $\bar{c}=\sqrt{8 R T / \pi}$

$c_{v} \quad$ Specific heat at constant volume

E Energy

$F \quad$ Velocity distribution function

$J \quad$ Flux

$k \quad$ Boltzmann constant, $1.380658 \times 10^{-23} J^{-1}$

l Unit normal

$M \quad$ Mach number

$\mathcal{M} \quad$ Maxwellian distribution function

$m \quad$ Molecular mass

$N \quad$ Number of particles in a cell

$n \quad$ Particle number density

P Pressure tensor

$\mathcal{P} \quad$ Diagonal pressure tensor

$R \quad$ Specific gas constant

$s \quad$ Speed ratio

$T \quad$ Temperature

$t \quad$ Time
\end{abstract}

\footnotetext{
*Graduate Student Research Assistant, AIAA Student Member, E-mail: aerowwl@engin.umich.edu

${ }^{\dagger}$ Professor, AIAA Associate Fellow, E-mail: iainboyd@engin.umich.edu

Copyright (C) 2003 by the American Institute of Aeronautics and Astronautics, Inc. All rights reserved.
}

\begin{tabular}{|c|c|}
\hline $\mathbf{u}$ & Molecular macroscopic velocity \\
\hline $\mathbf{v}$ & Molecular microscopic velocity \\
\hline $\mathbf{x}$ & Coordinate system \\
\hline$\beta$ & $\begin{array}{l}\text { Reciprocal of the most probable molecular } \\
\text { thermal speed, } \beta=1 / \sqrt{2 R T}\end{array}$ \\
\hline$\rho$ & Mass density \\
\hline$\xi$ & Preserved macroscopic velocity \\
\hline$\zeta$ & Number of internal degrees of freedom \\
\hline
\end{tabular}

$\begin{array}{ll}\text { Subscripts } \\ 1 & \text { Upstream } \\ 2 & \text { Downstream } \\ c & \text { Cell average } \\ e & \text { Energy flux } \\ j & \text { Particle index } \\ m & \text { Mass flux }\end{array}$

\section{Introduction}

Over the past few decades, the direct simulation Monte Carlo (DSMC) method ${ }^{1}$ has been widely employed to solve rarefied gas flow problems in a variety of applications, such as hypersonic atmospheric re-entry flows and micro-electro-mechanical system (MEMS) problems. While the DSMC technique can be considered successful for the former problems, there are many challenges for the latter. The major challenge comes from the difficulty to effectively reduce the statistical scatter encountered in micro-scale flows. Unlike hypersonic flows, MEMS flows always have very large noise-to-signal ratio, which makes DSMC simulations require sample sizes over millions or even tens of millions before acceptable solutions can be obtained. This causes the DSMC approach to become extremely time-consuming in predicting MEMS flows.

In addition, it is prohibitively expensive for DSMC to simulate the continuum regime in which computational fluid dynamics (CFD) methods solving the Navier-Stokes (NS) equations are very effective. When combined with CFD methods to form a hybrid method that is computationally efficient and physically accurate, the DSMC technique needs to communicate information across the boundary between the CFD and DSMC domains. Once again, because of its statistical 
fluctuations, the DSMC method always needs several steps of sampling. Hybrid codes in this fashion are considered as weakly coupled ${ }^{2,3}$ and are inadequate for complex nonequilibrium flows.

To overcome the statistical scatter problem in the DSMC method, Fan and others developed an information preservation (IP) technique for low speed rarefied gas flows. ${ }^{4-7}$ In addition to the ordinary thermal velocity that is utilized to compute the particle trajectory, each simulation particle in the IP technique also possesses macroscopic preserved information such as velocity vector and temperature. Numerical experiments ${ }^{6-8}$ showed that the IP method works quite well in solving micro-scale gas flows.

In the most recent work by Sun and Boyd, ${ }^{9}$ an additional temperature term is introduced in the IP energy model to solve the contradiction between the real energy flux and the DSMC-IP representation of the translational energy flux across a cell interface. From gas kinetic theory, the volume averaged translational energy of a monatomic particle at equilibrium temperature $T$ is $3 k T / 2$ ( $k$ is the Boltzmann constant), whereas the average translational energy carried by a monatomic particle across an interface at equilibrium is $2 k T$. The extra energy, $k T / 2$ must be taken into account or the energy of the whole system will not be conserved. Therefore, each simulation particle in the IP method has an additional temperature $T_{a}$.

The additional temperature in the IP method of Ref. 9 is actually evaluated as $k\left(T-T_{\text {ref }}\right) / 2$ by assuming that there is a counterpart particle at temperature $T^{\prime}$ moving in the opposite direction and carrying extra energy $k\left(T^{\prime}-T_{r e f}\right) / 2$ across the same interface. Here, $T_{r e f}$ is a reference temperature and is very close to the preserved temperature $T$ of the particle. ${ }^{9}$ It has been numerically demonstrated that this IP method performs excellently for flows in which nonequilibrium is insignificant, such as Couette flow, Rayleigh flow, Mach 0.8 airflow over a NACA0012 airfoil with a chord length of $4 \mathrm{~cm}^{9}$ and Mach 0.2 airflow over a 20-micronlong flat plate. ${ }^{10}$ However, a numerical experiment of a supersonic flow over a $2-\mathrm{D}$ wedge ${ }^{11}$ showed that this IP method does not work very well near the leading edge and within the shock, where the flow is believed to be strongly nonequilibrium.

As Mach number is increased to hypersonic conditions, the accuracy of the IP method becomes very poor. A typical example for a Mach 5 shock in argon is displayed in Fig. 1 in which the results obtained with the IP method are compared with DSMC results. The non-dimensional variable is defined as

$$
Q^{*}=\frac{Q-Q_{\mathrm{ref}}}{\left|Q_{2}-Q_{1}\right|}
$$

where the subscripts 1 and 2 represent upstream and downstream of the shock, respectively. $Q_{\text {ref }}$ is $\rho_{1}$ and $T_{1}$ for density and temperature and $u_{2}$ for velocity.

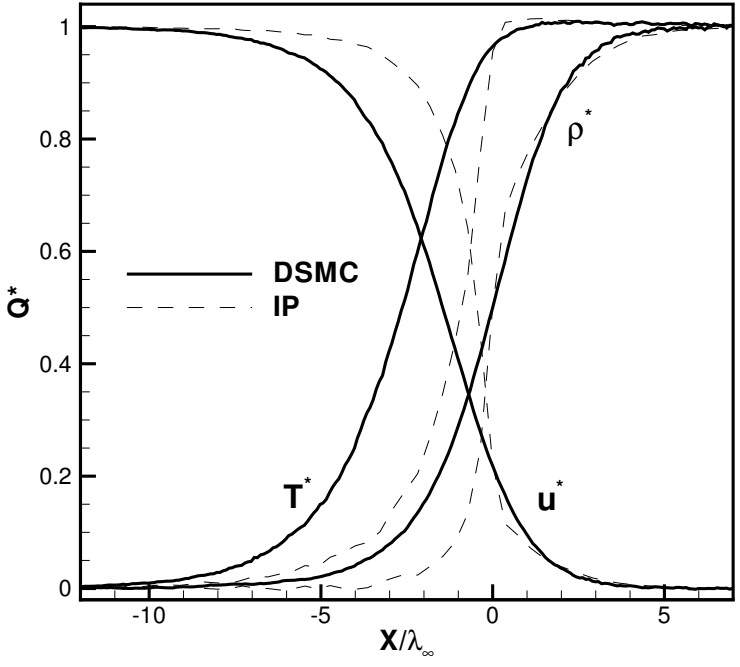

Fig. 1 Non-dimensional flow properties for a Mach 5 shock in argon.

It is clear that the IP method is unable to accurately predict the profile of any of the flow properties. Additionally, because the IP results fail to predict the onset of nonequilibrium at the upstream edge of the shock, the domain interface in a hybrid DSMC/CFD simulation is placed at the wrong location. As a result, the particle domain is always too small. The poor performance is really no surprise because the idea of how the IP approach deals with the energy carried by simulation particles from cell to cell is based on the assumption of local thermal equilibrium or weak nonequilibrium.

The main objective of the present study is therefore to focus on the improvement of the energy flux modeling in the IP method. A more general model that relaxes the local thermal equilibrium assumption will be described in the next section. Following that, the new model will be applied to a large range of Mach number in different gases. Detailed comparisons of shock wave structure are presented with other numerical methods and experimental data. Conclusions and suggestions for future work are provided in the last section.

\section{Numerical Scheme}

The time evolution in phase space of the particle velocity distribution function $F(\mathbf{x}, \mathbf{v}, t)$ of a dilute gas is governed by the Boltzmann equation and can be expressed in terms of time $t \in \mathbb{R}^{+}$, the spatial coordinates $\mathbf{x} \in \mathbb{R}^{3}$, and the molecular velocity $\mathbf{v} \in \mathbb{R}^{3}$. If external forces are absent, the Boltzmann equation reads

$$
\frac{\partial}{\partial t} F+\mathbf{v} \cdot \nabla F=\frac{\delta}{\delta t} F,
$$

subject to the initial condition

$$
F(\mathbf{x}, \mathbf{v}, t=0)=F_{0}(\mathbf{x}, \mathbf{v}) .
$$


A domain without any walls is considered in the present study. The right-hand side of Eq.1, $\delta F / \delta t$, is the collision operator and represents the time rate of change of the distribution function due to binary intermolecular collisions.

Assuming that the distribution function is normalized to the particle number density, $n$, if $Q(\mathbf{v})$ is a function of $\mathbf{v}$ but not of position and time, the moment of the distribution function $F$ with respect to $Q$ over the velocity space is defined as

$$
n \bar{Q}=\int_{\mathbb{R}^{3}} Q F d v .
$$

Consider a simple gas with molecular mass $m$ and number of internal degrees of freedom $\zeta$. Macroscopic variables of the gas such as density, velocity and temperature are moments of $F$

$$
\begin{aligned}
\rho & =\int_{\mathbb{R}^{3}} m F d v \\
\mathbf{u} & =\frac{1}{\rho} \int_{\mathbb{R}^{3}} m \mathbf{v} F d v \\
T & =\frac{1}{3 \rho R} \int_{\mathbb{R}^{3}} m(\mathbf{v}-\mathbf{u})^{2} F d v
\end{aligned}
$$

where $R$ is the specific gas constant. Furthermore, the conservation equations of the gas can be obtained by taking moments of the Boltzmann equation with respect to the collisional invariants $m, m \mathbf{v}$ and $m\left(v^{2}+\zeta R T\right) / 2$ (the principle of energy equipartition is applied),

$$
\begin{aligned}
& \frac{\partial}{\partial t} \rho+\nabla \cdot(\rho \overline{\mathbf{v}})=0 \\
& \frac{\partial}{\partial t} \rho \overline{\mathbf{v}}+\nabla \cdot(\rho \overline{\mathbf{v} \mathbf{v}})=0 \\
& \frac{\partial}{\partial t} \rho \bar{E}+\nabla \cdot\left(\frac{1}{2} \rho \overline{\mathbf{v}\left(v^{2}+\zeta R T\right)}\right)=0
\end{aligned}
$$

where $\bar{E}=\frac{1}{2} \overline{v^{2}+\zeta R T}$.

A simulation particle in the DSMC-IP method has not only the ordinary thermal velocity $\mathbf{v}$ but also preserved macroscopic velocity $\xi$ and temperature $T$. The change of the preserved velocity is proportional to the gradient of the scalar pressure for low speed flows. ${ }^{9,12}$ However, the pressure in strong shock waves is no longer in equilibrium in all directions. A more general pressure gradient term is involved

$$
\frac{\partial \xi}{\partial t}=-\frac{1}{\rho} \nabla \cdot \mathcal{P}
$$

where $\mathcal{P}$ consists of only the diagonal elements of the pressure tensor

$$
\mathbf{P}=m \int_{\mathbb{R}^{3}}(\mathbf{v}-\mathbf{u})(\mathbf{v}-\mathbf{u}) F d v .
$$

Since a simulation particle $j$ represents a large number of real molecules, it is reasonable to assume that the real molecules are in some kind of velocity distribution characterized by $\xi_{j}$ and $T_{j}$. In the present study, the distribution is assumed to be Maxwellian

$$
\mathcal{M}_{j}=\frac{1}{\left(2 \pi R T_{j}\right)^{3 / 2}} \exp \left(-\frac{\left|\mathbf{v}-\xi_{j}\right|^{2}}{2 R T_{j}}\right) .
$$

The local velocity distribution function within a cell with $N$ simulation particles is then assumed to be the arithmetic average of all $\mathcal{M}_{j}$ in the cell

$$
F_{c}=\frac{n}{N} \sum_{j=1}^{N} \mathcal{M}_{j}
$$

Notice that it is not necessary for the $F_{c}$ to be Maxwellian. For the sake of simplicity, summation always means from $j=1$ to $N$ and its upper and lower limits are dropped from now on.

With the above assumption of the local velocity distribution function in a cell, cell velocity and temperature can be determined using Eqs. 3 and 4, respectively, and are

$$
\begin{aligned}
\mathbf{u}_{c} & =\frac{1}{N} \sum \xi_{j}, \\
T_{c} & =\frac{1}{N} \sum \frac{1}{3 R}\left|\xi_{j}-\mathbf{u}_{c}\right|^{2}+T_{j} .
\end{aligned}
$$

Notice that $T_{c}$ is always positive as long as every particle in the cell has a positive IP temperature. The diagonal pressure tensor in Eq. 8 has elements

$$
\mathcal{P}_{i k}=\delta_{i k} \frac{\rho}{N} \sum\left(\xi_{j, i}-u_{c, i}\right)\left(\xi_{j, k}-u_{c, k}\right)+R T_{j}
$$

where $\delta_{i k}$ is the Kronecker delta.

To solve the continuity and energy equations (Eqs. 5 and 7 , respectively), mass and energy fluxes across a cell interface must be evaluated. A general expression of the flux of $Q(\mathbf{v})$ across a cell interface can be written as

$$
J_{Q}=\int_{\mathbb{R}^{2} \times \mathbb{R}^{+}} v_{n} Q F_{c} d v
$$

where $v_{n}$ is the velocity component in the direction normal to the cell interface and is defined as $v_{n}=\mathbf{v} \cdot \mathbf{l}$ where $\mathbf{l}$ is the unit normal to the interface. One integration in Eq. 13 has been limited to those molecules moving in the positive l-direction. Mass and energy fluxes can be obtained when $Q$ in Eq. 13 is replaced with $m$ and $m\left(v^{2}+\zeta R T\right) / 2$. With the help of Eqs. 9 and 10, the fluxes can be evaluated as

$$
\begin{gathered}
J_{m}=\frac{1}{N} \sum J_{m, j}, \\
J_{e}=\frac{1}{N} \sum J_{m, j}\left[\xi_{j}^{2}+(5+\zeta) R T_{j}\right] / 2- \\
\rho_{c} R T_{j} \bar{c}_{j} \exp \left(-s_{j}^{2}\right) / 8 .
\end{gathered}
$$

Here, $J_{m, j}$ is the mass flux resulting from a simulation particle $j$ and reads

$$
J_{m, j}=\frac{1}{2} \rho_{c} \beta_{j}^{-1}\left\{s_{j}\left[1+\operatorname{erf}\left(s_{j}\right)\right]+\exp \left(-s_{j}^{2}\right) / \sqrt{\pi}\right\},
$$


where $s_{j}=\beta_{j} u_{n, j}$ is the speed ratio, $\beta_{j}^{-1}=\sqrt{2 R T_{j}}$ and $\operatorname{erf}(x)$ is the error function. The IP velocity component in the direction normal to the interface is denoted by $u_{n, j}=\xi_{j} \cdot$ l. $\bar{c}_{j}=\sqrt{8 R T_{j} / \pi}$ is the average thermal speed.

The rates of change of the density and average energy in a cell are approximated using the surface integrals with respect to the mass and energy fluxes around the cell boundary $\Omega$

$$
\begin{aligned}
& \frac{\partial}{\partial t} \rho_{c}=-\frac{1}{\Delta V} \oint_{\Omega} J_{m} d A, \\
& \frac{\partial}{\partial t} \rho_{c} E_{c}=-\frac{1}{\Delta V} \oint_{\Omega} J_{e} d A,
\end{aligned}
$$

where $\Delta V$ is the cell volume. A net flux consists of the outlet flux from the cell and the inlet flux from the neighboring cell.

A similar approach was proposed in the Equilibrium Flux Method (EFM) by Pullin, ${ }^{13}$ but there were no particles involved and all fluxes were evaluated based on cell averaged values. Shen et al. ${ }^{14}$ attempted to calculate the energy flux in a fashion close to that described here, but they assumed that all particles within a cell have the same preserved information.

Particle IP temperature is updated by assuming that all particles within a cell bear the same amount of temperature change

$$
\Delta T=\left\{E_{c}-\frac{1}{N} \sum\left(\frac{1}{2} \xi_{j}^{2}+c_{v} T_{j}\right)\right\} / c_{v},
$$

where $c_{v}=(3+\zeta) R / 2$. If $\Delta T$ is positive, particle temperature in the cell increases and everything is well defined. However, when $\Delta T$ is negative, care must be taken to prevent the particle IP temperature from being negative or lower than a cut-off temperature $T_{\theta}$, therefore a loop process is employed to update the particle temperature. The loop process starts with a test of each individual particle in a cell to examine whether the particle IP temperature is lower than $T_{\theta}$ when $\Delta T$ is applied. If the test is false, the temperature update for the individual particle is allowed. Otherwise, the temperature update is not allowed and an amount of $\Delta T$ is banked. After all particles in the cell are finished with the update for a loop, the banked temperature is divided by the number of particles in the cell and the result is a new $\Delta T$ whose absolute value is less than the last one. The loop repeats with the new $\Delta T$ until there is no banked temperature. From the experience of numerical experiments, the number of loops is always smaller than $50 . T_{\theta}$ is arbitrarily chosen to be $5 \%$ of the local cell temperature.

\section{Numerical Examples}

The current DSMC-IP code is based on a parallel optimized DSMC code named MONACO. ${ }^{15}$ A sub-cell scheme is implemented for selection of collision pairs where the number of sub-cells is scaled by the local mean free path.

The new energy flux model is evaluated using 1-D stationary shock wave simulations. A computational domain that is sufficiently large to contain the shock is employed. Particles entering the computational domain from the upstream side are initialized with the upstream conditions. A moving piston boundary described in the book by $\operatorname{Bird}^{1}$ is applied at the downstream edge. IP properties of the particles reflecting from the moving piston back to the computational domain are set with the downstream conditions.

For both pure DSMC and DSMC-IP simulations, steady state for each case considered below is assumed to be reached after the flow has moved approximately 20 shock widths. After that, dependent on the Mach number, another 20,000 to 100,000 time steps are taken to sample the results for the pure DSMC simulations. A much smaller sampling time of 1,000 steps is taken for all the DSMC-IP calculations.

\section{Shocks in Argon}

The first type of gas of interest in the present numerical investigation is argon. The upstream conditions are: $T_{1}=300 \mathrm{~K}, \rho_{1}=1.068 \times 10^{-4} \mathrm{~kg} / \mathrm{m}^{3}$ and $a_{1}=322.6 \mathrm{~m} / \mathrm{s}$. Unless otherwise specified, the computational domain spans about 40 upstream mean free paths and is evenly divided between the upstream and downstream regions with the initial discontinuity at the origin. Although this is a 1-D problem, a 2-D grid with 400 uniform cells in the flow direction and 5 cells in the transverse direction is employed. The results shown below are from the middle 400 cells. The number of particles per cell in the upstream is about 30 on average. The reference time-step is $10 \mathrm{nsec}$ in all cases.

In Fig. 2, results from the present IP method for a weak Mach 1.55 shock are compared with the old IP $\operatorname{method}^{9}$ and with DSMC. It is clear that the present IP method is better than the old one. Although the profiles of the present IP method and the DSMC in the figure are in good agreement in the rear portion of the shock, the present method still fails to predict the onset of temperature increase at the upstream edge of the shock. Detailed inspection reveals that the density profile calculated with the current IP method is slightly thinner.

Figure 3 shows the results as Mach number is increased to 4 . The density profile predicted with the present IP method is in outstanding agreement with the DSMC results. The discrepancies of the temperature and velocity at the shock front still persist but they do not look any worse than in the last example at lower Mach number. On the other hand, the old IP method results look very poor.

Under hypersonic upstream conditions, it is very difficult for the old IP method to generate reasonable 


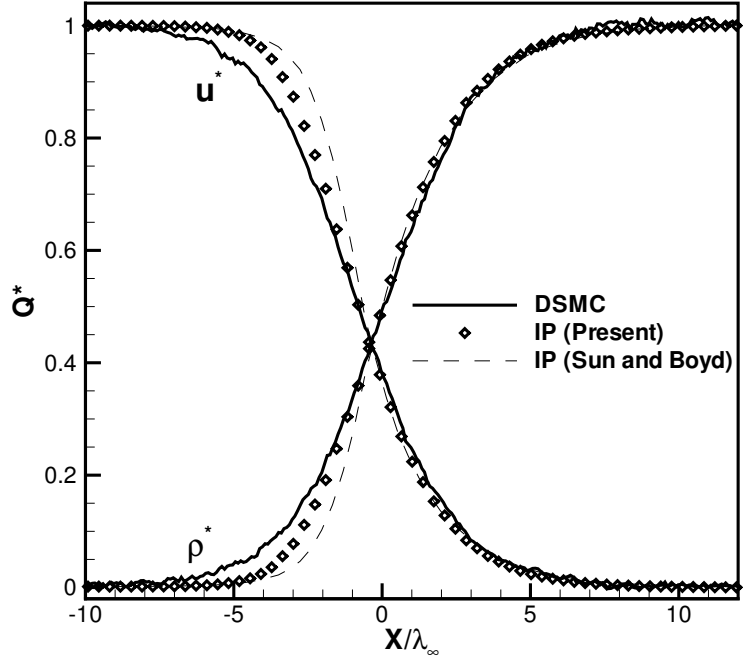

a) Comparisons for density and velocity.

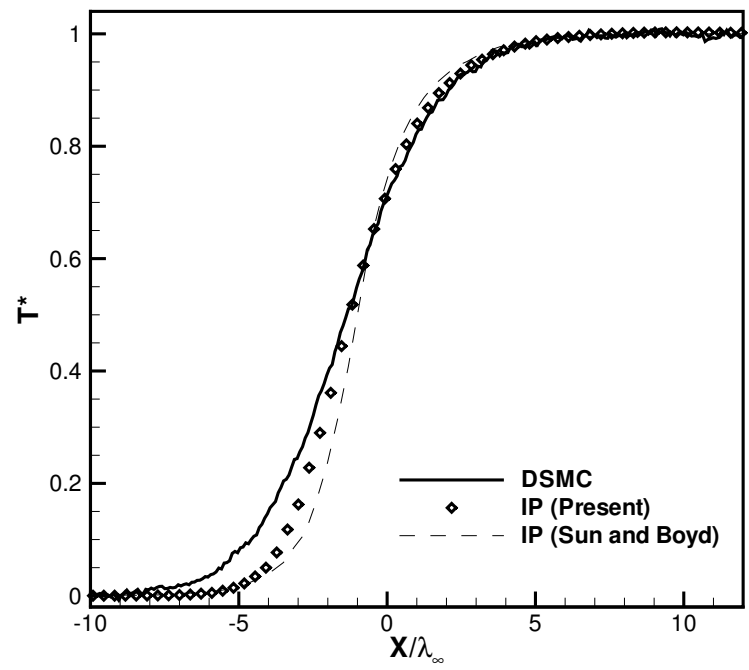

b) Comparisons for temperature.

Fig. 2 Comparisons for non-dimensional flow properties for a Mach 1.55 shock in argon.

results at Mach numbers higher than 5. To emphasize the great improvement by the new energy flux model, the comparisons for the current and the old IP methods and DSMC at Mach 5 are made in Fig. 4. Obviously, the new method is much better than the old one.

As Mach number is increased to 8, the IP method with the new energy flux model also works well. Comparisons for non-dimensional density, temperature and velocity of the IP and DSMC methods are displayed in Fig. 5. The density profile predicted with the IP method is again in excellent agreement with the DSMC results although the IP method predicts a slightly thicker shock.

The size of the downstream computational region at this Mach number and higher is increased to about 60 upstream mean free paths. Numerical experiments demonstrated mismatched downstream boundary con-

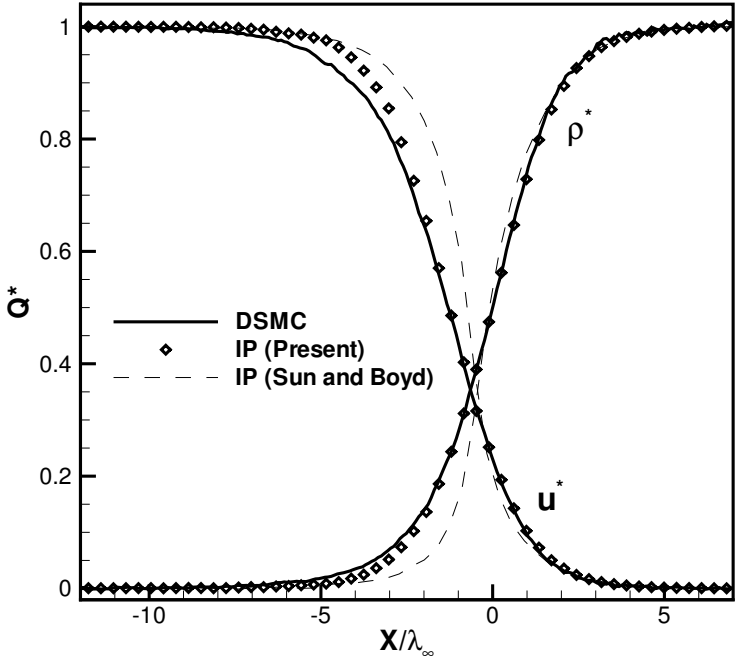

a) Comparisons for density and velocity.

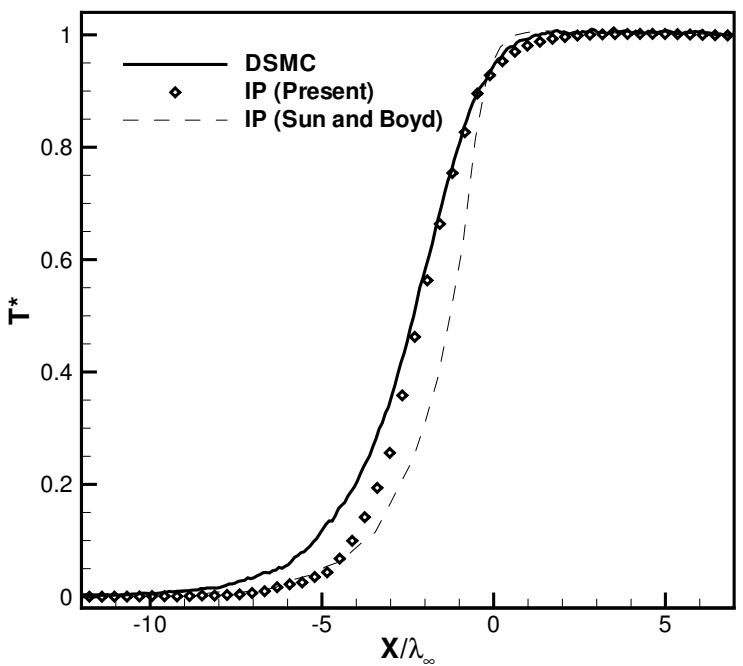

b) Comparisons for temperature.

Fig. 3 Comparisons for non-dimensional flow properties for a Mach 4 shock in argon.

ditions if the size of the downstream region is too small.

The reciprocal shock thickness (a measure of the maximum density gradient) is shown in Fig. 6 as a function of Mach number. The experimental data are from Alsmeyer ${ }^{16}$ and Navier-Stokes data from Boyd. ${ }^{17}$ The most striking conclusion that can be made is that the current IP method predicts shock density profiles much closer to the experimental and DSMC results for all Mach numbers than the old IP method. When Mach number is very high, the shock thickness calculated with the IP method is slightly thicker than the measured data. The overall accuracy of the new IP method is much better than the CFD method, except at very low Mach numbers. Notice that the old IP method stops at Mach 5 because reasonable results are difficult to obtain for higher Mach numbers. 


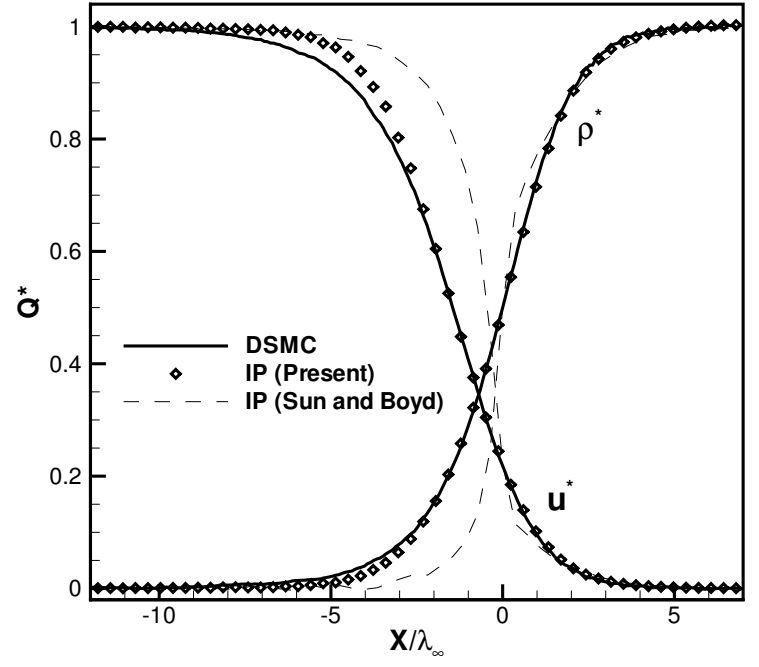

a) Comparisons for density and velocity.

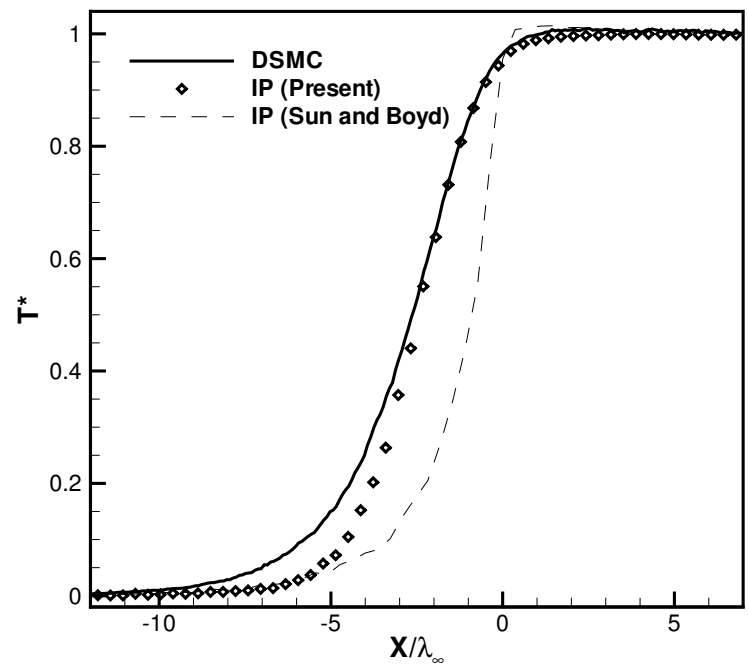

b) Comparisons for temperature.

Fig. 4 Comparisons for non-dimensional flow properties for a Mach 5 shock in argon.

\section{Shocks in Nitrogen}

The next example is a Mach 5 shock in nitrogen, which has $\zeta=2$ due to rotation. The upstream conditions are: $u_{1}=1248.6 \mathrm{~m} / \mathrm{s}, T_{1}=300 \mathrm{~K}$ and $\rho_{1}=7.483 \times 10^{-5} \mathrm{~kg} / \mathrm{m}^{3}$. The downstream temperature is $T_{2}=1740 \mathrm{~K}$. The computational domain spans about 50 upstream mean free paths, otherwise it is the same as in the previous examples. A similar 2-D grid is again employed. The number of particles per cell in the upstream is also about 30 . The reference time-step is $20 \mathrm{nsec}$.

The results from the new IP method are in very good agreement with the DSMC results in terms of density and velocity, as shown in Fig. 7(a). The reciprocal shock thickness predicted by DSMC and the new IP method is 0.345 and 0.324 , respectively, compared with the measured data of about 0.35 from Alsmeyer. ${ }^{16}$

An overall kinetic temperature $T_{\mathrm{OV}}$ may be intro-

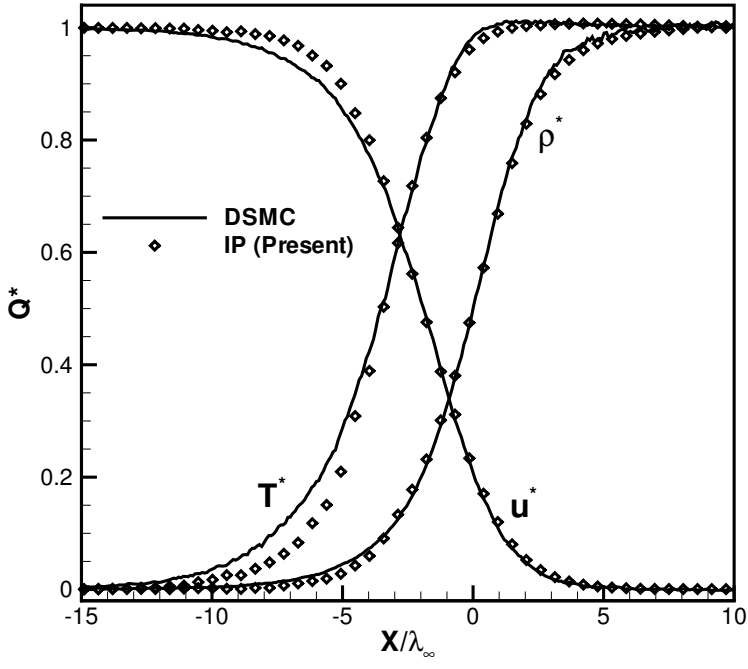

Fig. 5 Comparisons for non-dimensional flow properties for a Mach 8 shock in argon.

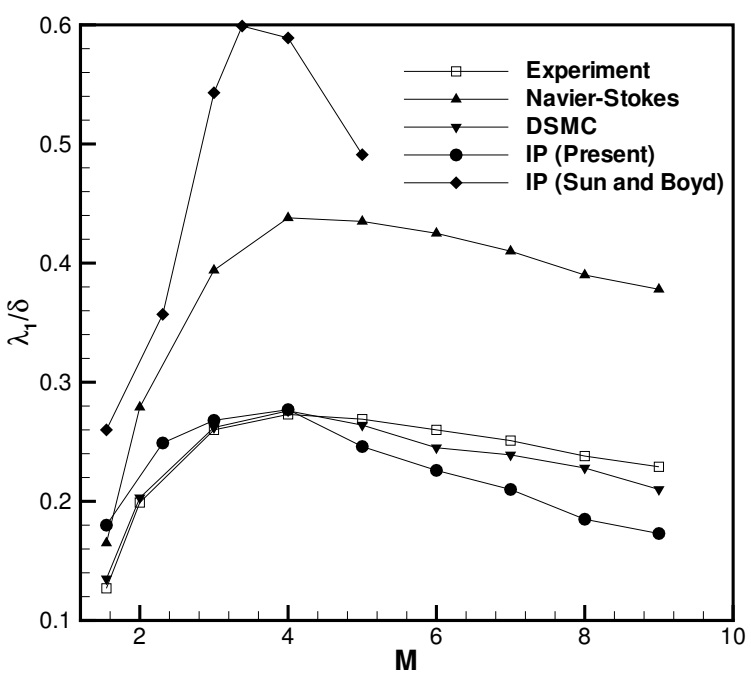

Fig. 6 Comparisons for reciprocal shock thickness for shock waves in argon.

duced for a nonequilibrium gas as the weighted average of the translational and internal temperatures ${ }^{1}$

$$
T_{\mathrm{OV}}=\left(3 T_{\text {tra }}+\zeta T_{\text {int }}\right) /(3+\zeta) .
$$

Strong nonequilibrium effects can easily be observed from the significant differences between the DSMC translational and rotational temperatures in Fig. 7(b).

Because the principle of equipartition of energy is applied in the current IP method, meaning that the translational and internal temperatures must be equal, there is no way at this point for the IP method to predict this kind of nonequilibrium. This will be discussed in the future work. Unlike the argon gas examples, the onset of temperature at the upstream edge of the Mach 5 nitrogen shock is well captured by the IP method. The resulting IP temperature profile also shows a $0.8 \%$ overshoot near the center of the shock. Higher Mach numbers are not considered because the downstream 


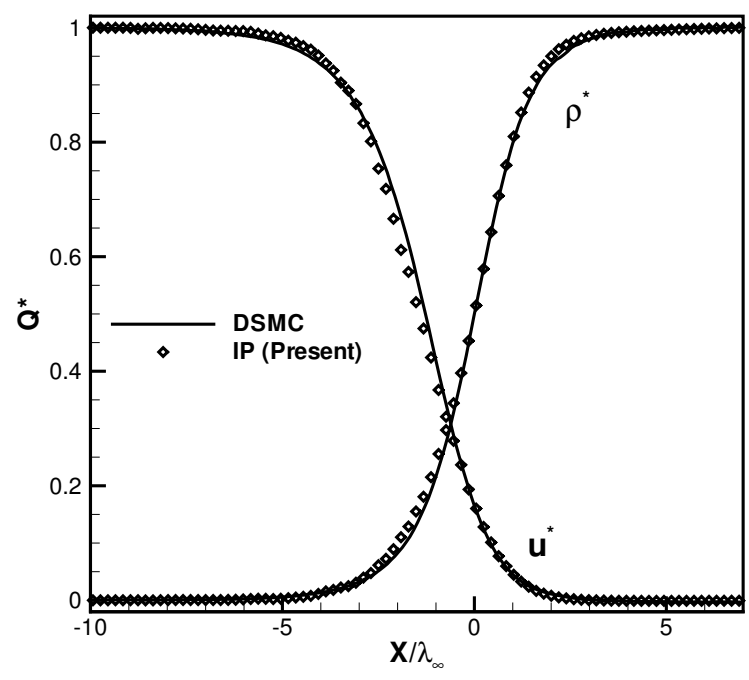

a) Comparisons for density and velocity.

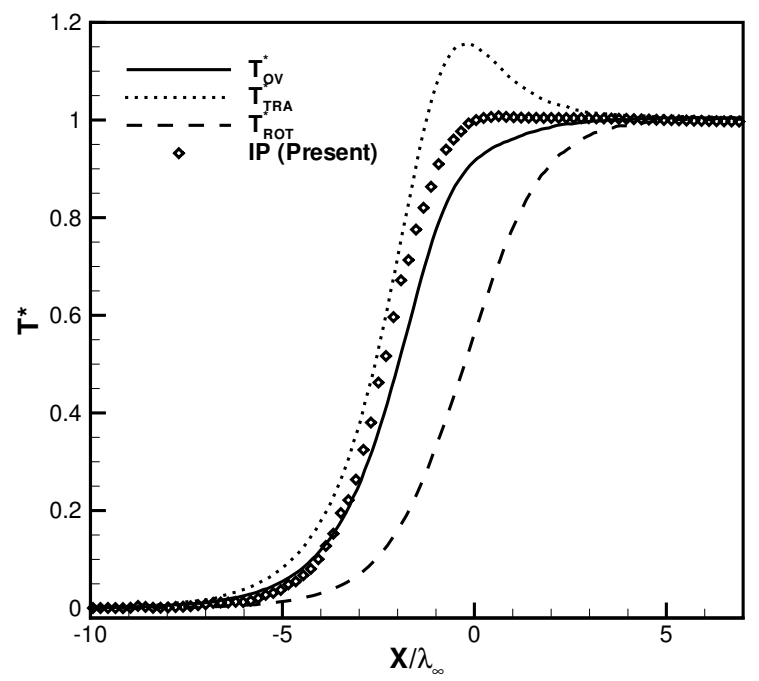

b) Comparisons for temperature.

Fig. 7 Comparisons for non-dimensional flow properties for a Mach 5 shock in nitrogen.

temperature will increase substantially. At Mach 6, for instance, the downstream temperature increases to about $2,400 \mathrm{~K}$, and it is difficult to judge at such high temperature the accuracy of the new IP method due to the nonequilibrium effects.

\section{Concluding Remarks and Future Work}

A new energy flux model has been developed to relax the local thermal equilibrium assumption in the Information Preservation method and improve its accuracy in shock waves, where the flows are strongly nonequilibrium. A simulation particle in the IP method possesses not only the microscopic thermal velocity but also a macroscopic preserved velocity and temperature. The new model was constructed using the assumption that the real molecules represented by a particle follow the Maxwellian distribution characterized by the preserved velocity and temperature and the local velocity distribution in a cell is the average of all particles within the cell.

The new energy flux model was tested for simulations of normal shock waves in argon over a wide range of Mach numbers and in nitrogen at Mach 5. It is concluded that results from the IP method incorporating the new model are in good agreement compared with the standard DSMC results and experimental data.

The main concern of the new model is that it is not capable of accurately predicting the onset of temperature rise at the shock front. This indicates that the particle domain in a hybrid DSMC/CFD simulation will still be too small if the domain interface is determined based on the IP information.

In addition to the temperature onset concern, there are three issues regarding the new model. First, numerical efficiency is very poor primarily because two time-consuming functions, exp and erf, are largely involved. Second, the model is sensitive to the number of particles per cell. Numerical experience suggests that it is required to have at least 30 particles in each cell. Combining these two issues implies that the current IP method is very expensive computationally. The third issue is about the downstream boundary conditions at Mach number higher than 8 . If the size of the downstream region is too small, the calculated post-shock state will be inconsistent with the imposed downstream boundary conditions. The post-shock density is always underestimated and temperature always overestimated. The reason for this is not yet understood.

The new energy flux model has been so far developed and tested in a domain without walls. The next step will be to develop or adopt a gas-surface model that can work along with the current model. A method modeling the nonequilibrium of the translational and internal temperatures is also necessary for non-monatomic gases in order to relax the equipartition assumption.

\section{Acknowledgments}

This work was sponsored by the Air Force Office of Scientific Research under grant F49620-01-1-0003. The views and conclusions contained herein are those of the authors and should not be interpreted as necessarily representing the official policies or endorsements, either expressed or implied, of the AFOSR or the U.S. Government.

\section{References}

\footnotetext{
${ }^{1}$ Bird, G. A., Molecular Gas Dynamics and the Direct Simulation of Gas Flows, Oxford University Press, Oxford, 1994.

${ }^{2}$ Rovedo, R., Goldstein, D. B., and Varghese, P. L., "Hybrid Euler/Particle Approach for Continuum/Rarefied Flows," Journal of Spacecraft and Rockets, Vol. 35, No. 3, 1998, pp. 258-265.

${ }^{3}$ Hash, D. B. and Hassan, H. A., "Assessment of Schemes for Coupling Monte Carlo and Navier-Stokes Solution Methods," Journal of Thermophysics and Heat Transfer, Vol. 10, No. 2, 1996, pp. 242-249.
} 
${ }^{4}$ Fan, J. and Shen, C., "Statistical Simulation of LowSpeed Unidirectional Flow in Transition Regime," Proceedings of the 21th International Symposium on Rarefied Gas Dynamics, Marseille, France, edited by e. a. R. Brum, 1998, p. 245.

${ }^{5}$ Fan, J. and Shen, C., "Statistical Simulation of LowSpeed Rarefied Gas Flows," Journal of Computational Physics, Vol. 167, No. 2, 2001, pp. 393-412.

${ }^{6}$ Cai, C., Boyd, I. D., Fan, J., and Candler, G. V., "Direct Simulation Methods for Low-Speed Microchannel Flows," Journal of Thermophysics and Heat Transfer, Vol. 14, No. 3, 2000, pp. 368-378.

${ }^{7}$ Sun, Q., Boyd, I. D., and Candler, G. V., "Numerical Simulation of Gas Flow Over Micro-Scale Airfoils," Journal of Thermophysics and Heat Transfer, Vol. 16, No. 2, 2002, pp. 171-179.

${ }^{8}$ Fan, J., Boyd, I. D., Cai, C.-P., Hennighausen, K., and Candler, G. V., "Computation of Rarefied Gas Flows Around a NACA 0012 Airfoil," AIAA Journal, Vol. 39, 2001, pp. 618-625.

${ }^{9}$ Sun, Q. and Boyd, I. D., "A Direct Simulation Method for Subsonic, Micro-Scale Gas Flows," Journal of Computational Physics, Vol. 179, No. 2, 2002, pp. 400-425.

${ }^{10}$ Sun, Q., Boyd, I. D., and Candler, G. V., "A Hybrid Continuum/Particle Approach for Micro-Scale Gas Flows," Proceedings of the 23th International Symposium on Rarefied Gas Dynamics, Wistler, Canada, edited by A. D. Ketsdever and E. P. Muntz, 2003, pp. 752-759.

${ }^{11}$ Wang, W.-L., Sun, Q., and Boyd, I. D., "Assessment of a Hybrid Method for Hypersonic Flows," Proceedings of the 23th International Symposium on Rarefied Gas Dynamics, Wistler, Canada, edited by A. D. Ketsdever and E. P. Muntz, 2003, pp. 923-930.

${ }^{12}$ Sun, Q., Information Preservation Methods for Modeling Micro-Scale Gas Flows, Ph.D. thesis, Department of Aerospace Engineering, University of Michigan, 2003.

${ }^{13}$ Pullin, D. I., "Direct Simulation Methods for Compressible Inviscid Idal-Gas Flow," Journal of Computational Physics, Vol. 34, 1980, pp. 231-244.

${ }^{14}$ Shen, C., Jiang, J. Z., and Fan, J., "Information Preservation Method For The Case of Temperature Variation," Proceedings of the 22nd International Symposium on Rarefied Gas Dynamics, Sydney, Australia, edited by T. J. Bartel and M. A. Gallis, Vol. 585, 2001, pp. 185-192.

${ }^{15}$ Dietrich, S. and Boyd, I. D., "Scalar and Parallel Optimized Implementation of the Direct Simulation Monte Carlo Method," Journal of Computational Physics, Vol. 126, 1996, pp. 328-342.

${ }^{16}$ Alsmeyer, H., "Density Profiles in Argon and Nitrogen Shock Waves Measured by the Absorption of an Electron Beam," Journal of Fluid Mechanics, Vol. 74, No. 3, 1976, pp. $497-513$.

${ }^{17}$ Boyd, I. D., "Predicting Breakdown of the Continuum Equations Under Rarefied Flow Conditions," Proceedings of the 23th International Symposium on Rarefied Gas Dynamics, Wistler, Canada, edited by A. D. Ketsdever and E. P. Muntz, 2003, pp. 899-906. 